\title{
De la Degradación y la Rehabilitación: Apuntes Conceptuales en torno a la Noción de Barrio
}

\author{
Degradation and Rehabilitation: Conceptual Notes on the Notion of the \\ Neighborhood
}

Degradação e Reabilitação: Notas Conceituais sobre a Noção de Bairro

\author{
Berenice Vizcarra \\ Doctora en Planeación y Desarrollo Sustentable \\ Universidad Autónoma de Baja California, México. \\ luz.vizcarra@uabc.edu.mx \\ (i) https://orcid.org/0000-0002-5984-0755
}

Recibido: junio 19 de 2021

Aceptado: julio 28 de 2021

Publicado: julio 30 de 2021

\section{RESUMEN}

El contemporáneo fenómeno de deterioro y abandono de los asentamientos habitacionales fuerza la implementación arbitraria de proyectos urbanos y arquitectónicos, que no siempre responden a las necesidades socioculturales de los habitantes. El siguiente artículo tiene el propósito de exponer un planteamiento teórico-conceptual, a partir del análisis y evaluación de los procesos de degradación y rehabilitación en los barrios habitacionales, apoyado en estudios sobre Planeación Urbana, Sociología Urbana y Desarrollo Sostenible. El resultado tiene la intención de ser un corpus referencial que auxilie la postulación de alternativas ante los crecientes casos de pérdida de Barrios con valor patrimonial.

Palabras clave: Barrio; degradación; rehabilitación; evolución urbana.

\begin{abstract}
The contemporary phenomenon of deterioration and abandonment of housing settlements forces the arbitrary implementation of urban and architectural projects, which do not always respond to the socio-cultural needs of the inhabitants. The following article aims to present a theoretical-conceptual approach, based on the analysis and evaluation of the degradation and rehabilitation processes in residential neighborhoods, supported by studies on Urban Planning, Urban Sociology and Sustainable Development. The result is intended to be a referential corpus that helps the application of alternatives in the face of increasing cases of loss of neighborhoods with patrimonial value.
\end{abstract}

Keywords: Neighborhood; degradation; rehabilitation; urban evolution.

\section{RESUMO}

O fenômeno contemporâneo de deterioração e abandono de loteamentos obriga a execução arbitrária de projetos urbanísticos e arquitetônicos, que nem sempre respondem às necessidades socioculturais dos 
moradores. O seguinte artigo tem como objetivo apresentar uma abordagem teórico-conceitual, baseada na análise e avaliação dos processos de degradação e reabilitação em bairros residenciais, apoiada em estudos de Planejamento Urbano, Sociologia Urbana e Desenvolvimento Sustentável. O resultado pretende ser um corpus referencial que auxilie na aplicação de alternativas face aos crescentes casos de perda de bairros com valor patrimonial.

Palavras-chave: Bairro; degradação; reabilitação; evolução urbana.

\section{INTRODUCCIÓN}

Debido al rápido y vertiginoso crecimiento urbano, un alto número de asentamientos habitacionales han comenzado un proceso de degradación, caracterizado por el desalojo de los inmuebles y el deterioro de los elementos físicos que los componen. Estas circunstancias no son exclusivas de una tipología, nivel socioeconómico o un área en particular dentro de las ciudades, puesto que la sobreproducción de viviendas ha causado el prematuro abandono de fraccionamientos y residenciales enteros en zonas indistintas de las metrópolis. Sin embargo, llega a ser preocupante cuando esta problemática se presenta dentro de los polígonos o centros fundacionales, donde barrios tradicionales se ven sustituidos por los nuevos conjuntos habitacionales en las periferias de las ciudades en expansión, atentando con la pérdida arquitectónica y social de elementos con valor histórico o patrimonial.

En cualquiera de los casos, las soluciones se han reducido a la precoz y arbitraria implementación de proyectos tanto arquitectónicos como urbanos, los cuales llegan a ignorar -en muchas de las ocasiones- el contexto social, histórico y cultural que existe en estos barrios, así como a desatender las necesidades de habitabilidad de la comunidad que aún permanece.

Este trabajo es un postulado teórico-conceptual que tiene como objetivo brindar una base referencial en la búsqueda de alternativas resilientes para este fenómeno urbano-social; resultado del análisis y evaluación de la espiral que conforman los procesos urbanos en áreas habitacionales, con base en estudios referentes a la planeación urbana y las teorías socioespaciales, de la mano con posturas como el desarrollo social sostenible y la teoría crítica. El escrito partirá del establecimiento de un marco teórico de interpretación que, consecuentemente, llevará a la definición de tres conceptos básicos para el estudio de la evolución urbana actual: barrio, degradación de barrios y rehabilitación de barrios.

\section{Procesos Urbanos desde la Planeación Urbano Sostenible: Un Marco de Interpretación}

Partiendo de la primicia de que los principales cambios y transformaciones de la sociedad se reflejan en el espacio urbano, este punto de vista obliga también a analizar el espacio en clave temporal para comprender las estructuras y formas, pasadas y presentes de la ciudad (Delgado, 2016, p. 119).

Toda constitución de un núcleo habitacional responde a un conjunto de etapas sucesivas consecuentes de los elementos políticos, económicos y sociales que se presentan en un determinado momento histórico, es decir, un proceso. De manera ordinaria, este proceso comienza con el establecimiento de un grupo de personas sobre un territorio; legislativamente a esto se le conoce como fundación. A partir de este hecho, da inicio la dotación de servicios básicos, el aumento de la densidad poblacional y la conformación de una dinámica social que le brinda carácter al lugar, entre otros aspectos que enmarcan la formación de la zona habitacional.

Cuando el territorio en formación alcanza una estabilidad en sus elementos físicos, económicos y sociales, se puede hablar de una etapa de consolidación dentro del proceso. En ocasiones dicho espacio urbanizado llega a deteriorarse y los vínculos sociales a mermarse, lo que implica una nueva etapa, a la que llamaremos degradación y desde la cual pueden plantearse dos respuestas, de acuerdo con las implicaciones políticas, económicas y sociales que se desarrollen alrededor del territorio.

Primera respuesta: el despojo; se presenta como una postura determinante y puede ser entendida como la pérdida de los elementos físicos e 
intangibles que constituyeron el espacio; en otras palabras, el final del proceso en ese territorio. Segunda respuesta: la rehabilitación; que opta por la capacidad de resiliencia, tanto arquitectónica como social, al intervenir sobre la fase de degradación. En cualquiera de los dos casos, se cuenta con la posibilidad de comenzar un nuevo proceso; lo que conlleva a postular la tesis de que todo núcleo habitacional está regido por ciclos de vida (Figura 1.).

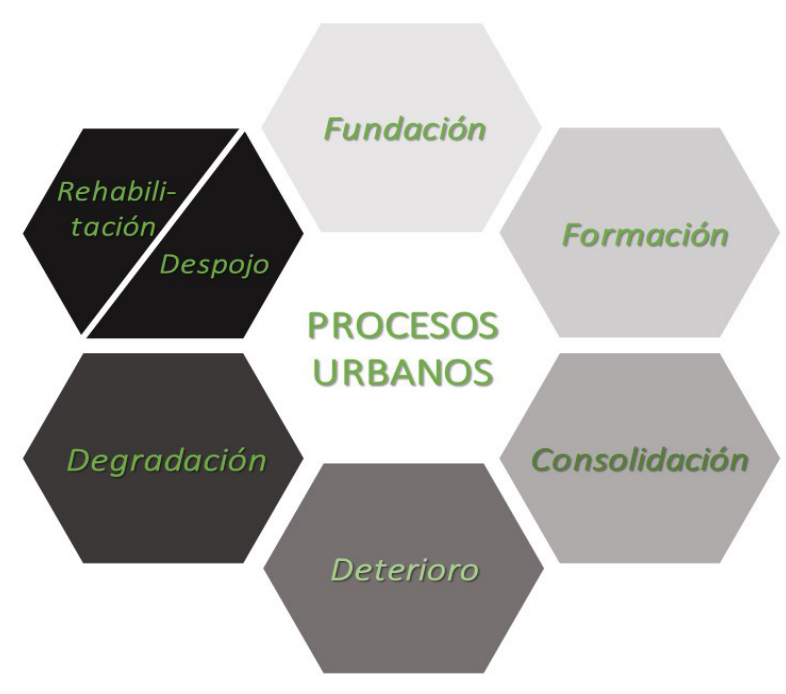

Figura 1. Diagrama de Procesos Urbanos.

Fuente: elaboración propia con base en información documental, 2021.

La inclusión de la dualidad entre degradación y rehabilitación barrial, como fases emergentes en el análisis de la evolución urbana, sugiere que estos procesos sean definidos e interpretados a partir de un entendimiento sobre la planeación urbano-sostenible, comprendiendo esto como el balance entre los elementos naturales, económicos y sociales del presente y para el futuro, en relación con el medio físico construido.

La planeación urbano-sostenible requiere de la visión, participación y acción interdisciplinaria, en busca de conectar las posibles soluciones a los diversos problemas de una ciudad. Siguiendo a Castro, "para que la planeación sea sostenible debe estar enfocada en aspectos sociales y ambientales [...] debe estar inscrita en un contexto estructurado y general, donde se tengan en cuenta factores e impactos en diversas escalas" (Castro, 2018, p. $66)$. En este sentido, la definición de los apuntes conceptuales para entender los procesos urbanos en los barrios, también se apoyará de las teorías socioespaciales, como la psicología ambiental y la sociología urbana, además de optar por una postura basada en la teoría crítica.

Hoy día, el desarrollo sostenible de las ciudades está lejos de conseguirse. Si bien autores como Rojas et. al. (2012) mencionan que se requiere la inclusión de lo político-institucional para lograr que la idea de desarrollo sostenible pueda instrumentarse como planeación-acción; la planeación urbana en América Latina resulta muchas de las veces ineficiente, mientras que los planes de desarrollo urbano tienen poco éxito en la práctica, en tanto que cumplen solamente una función de carácter normativo sin tener continuidad entre sí, pues, generalmente, proyectos y programas reinician con cada cambio de administración pública, lo que favorece a unos cuantos pero deja al margen las necesidades de la sociedad, de manera que los fenómenos urbanos avanzan y se alejan de una posible solución (Castro, 2018).

Al respecto, Giglia argumenta que "la planeación no es de ninguna manera un instrumento neutral [...] favorece los intereses de ciertos actores a expensas de otros, además de responder a las lógicas del mercado prevalecientes en ciertos momentos históricos" (Giglia, 2012, p. 82). Esto se revela con las numerosas investigaciones respecto a los procesos de gentrificación, procesos de despoblamiento y en estudios sobre desarrollo geográfico desigual.

Ante esto, también resulta importante enmarcar la injerencia del sentido de patrimonio dentro de la duplicidad de los procesos de degradación y rehabilitación en los asentamientos históricos; y es que es en esta etapa final de la evolución urbana donde pudieran verse perdidos los valores forjados por la comunidad barrial, de manera intencional o arbitraria. Entendamos a los valores patrimoniales como tangibles e intangibles; ya sean arquitectónicos, urbanísticos, sociales o culturales, se invita "a mirar los elementos patrimoniales [...] como rasgos de identidad y, simultáneamente, observar el deseo colectivo que llevó a encontrar su satisfacción en esos elementos." (Pérgolis y Quijano, 2020, p. 12) 
Choay asegura que "la noción de patrimonio urbano histórico se constituye a contracorriente del proceso de urbanización dominante" (Choay, 2019, p. 164); lo que, a decir de este tema, corresponde a la promoción de intervenciones en barrios en declive, sin la definición de qué elementos constituyen a un barrio en sí mismo, sin considerar las causas y manifestaciones de su deterioro y sin contemplar opciones que favorezcan a largo plazo.

Por lo anterior, la planificación de las ciudades necesita dejar de ser una actividad eminentemente reguladora y requiere transformarse en una gestión coordinada entre gobierno, sociedad e iniciativa privada, con el fin de concretar acciones sostenibles (Castro, 2018). Es entendible que para alcanzar este objetivo será concluyente generar un encuadre conceptual básico que apoye el dialogo entre los involucrados y que enmarque la definición de postulados.

Finalmente, al abordar el ámbito de la rehabilitación urbana, será prudente partir de las aportaciones teóricas de la sustentabilidad social. Al decir de Dave (2011), esta se puede estudiar a través de dos grandes categorías. Por un lado, la equidad social, que incluye el acceso a servicios y oportunidades; y, por el otro, la sustentabilidad de comunidades, la cual requiere de la percepción de seguridad y el apego hacia los vecindarios, de la interacción y participación de las personas en las actividades colectivas, así como la percepción de calidad y satisfacción tanto del hogar como del entorno. Este último grupo guarda mayor relación con el sentido de pertenencia y arraigo en comunicación con la estructura de la ciudad y, por tanto, se vincula con la planeación urbana sostenible.

\section{Aspectos Metodológicos}

Puesto que el propósito de este escrito es elaborar un corpus referencial que brinde un planteamiento teórico para la postulación de alternativas ante los crecientes casos de pérdida de asentamientos habitacionales con valor patrimonial, desde la definición de conceptos como barrio, degradación urbana y rehabilitación urbana; y no necesariamente implantar lineamientos concretos para un caso de estudio en particular; los métodos empleados para la elaboración de este artículo se limitaron a la revisión documental -particularmente bibliográfico- y al análisis inductivo; indirectamente apoyado de una filosofía empírica basada en años de recorridos de observación no participante en distintas ciudades latinoamericanas.
Tomando en consideración la interdisciplinariedad del trabajo, las fuentes se seleccionaron a partir de títulos clásicos y autores reconocidos en el área; además, se clasificaron de acuerdo con categorías derivadas de los ámbitos de estudio; es decir: la planeación urbana, la sociología urbana y el desarrollo sostenible.

En cuanto al análisis inductivo, se procuró llegar a una saturación de la información observable, hasta derivar en la hipótesis: la ausencia de aproximaciones teóricas y conceptuales sobre el contemporáneo fenómeno de deterioro y abandono de los asentamientos habitacionales con valor patrimonial, conlleva a la implementación arbitraria de proyectos urbanos y arquitectónicos, que no siempre responden a las necesidades socioculturales de los habitantes.

\section{Aproximaciones a la Noción de Barrio}

De manera ordinaria, se puede señalar que el barrio es una escala intermedia de organización social entre la vivienda y la ciudad, integrada por vialidades, edificaciones, infraestructura, espacios públicos, equipamiento, etcétera; las cuales constituyen su dimensión física. Sin embargo, al barrio no solo lo definen sus componentes físicos, sino la comunidad que lo forma, es decir, los intereses, similitudes o costumbres que se establecen de las redes sociales entre sus habitantes (Amérigo y Pérez, 2010).

Desde la sociología urbana se ha entendido el barrio como articulador entre las diversas escalas de la vida social urbana, integrador de la vida familiar, referente espacial, generador de identidad, articulador entre diversos grados de privacidad e integrador de las redes sociales de solidaridad y apoyo. (Rizo, 2006, p. 10)

Igualmente, desde la sociología urbana, Gravano habla del barrio como "una unidad colectiva 'consciente', de un nivel mayor que la unidad vecinal, con una 'personalidad' distintiva dentro de la ciudad, con límites definidos, con un nombre que no poseen los vecindarios- y con una 'cierta autonomía'" (Gravano, 2005, p. 99); y pretende decretar, igualmente, qué no lo es:

No es barrio, en primer lugar, el centro, usualmente confundido por connotación con el término ciudad. Luego, no es barrio la 
comunidad local, cuya existencia es previa a la expansión de la ciudad a partir de su centro. Y, en tercer término, no es barrio [...] aquella parte de la ciudad que no goza de estas características cualitativas. (Gravano, 2005, p. 100)

Mientras que "[Raymond Ledrut] percibe el barrio no como una unidad administrativa, sino como una realidad en el corazón de los procesos de estructuración y desestructuración sociológica. la diferenciación en el espacio social urbano la hacen efectivamente los barrios" (Lamy, 2006, p. 216). Por ello, a diferencia de otros apelativos para definir un núcleo habitacional -como lo serían una colonia, residencial, distrito o fraccionamiento, términos que hacen referencia a un módulo territorial delimitado por una institución catastral con el fin de facilitar la administración de estas en las ciudades-, el vocablo barrio nace de la necesidad de una denominación ante la particular interacción entre sociedad y espacio que se presentaba con la ciudad industrial del siglo XIX y que, hasta nuestros días, ha tomado forma a partir de la conjunción de las teorías sociales y de la planificación urbana.

Cabe anotar que, al no contar con las credenciales políticas que obtendría normalmente un distrito o una delegación a través de la sectorización administrativa, el barrio siempre quedará en la posición de resistencia, cuya organización y resolución de conflictos partirán de los intereses y problemáticas a las que se enfrente la propia comunidad.

Generalmente, un barrio está ligado a la simbolización de la población que lo conforma, pudiendo ser relativo a su procedencia, derivado del desplazamiento y posterior asentamiento de individuos categorizados por ciertas etnias o nacionalidades, relativo a su vocación que, a la plena usanza medieval, se determina por gremios, o relativo a su estatus social y económico. En cualquiera de las referencias, se establece al barrio como aquella unidad territorial con características imputadas por las dinámicas socioeconómicas y sus implicaciones en la movilidad rural-urbana y migratoria, en los oficios e ingresos, $\mathrm{o}$ en las posibilidades y opciones de adquisición de una vivienda, así como los servicios e infraestructura pública que aquí se presenten.

Al estudiar a Raymond Ledrut, Gravano (2005) comprende a los barrios como aquellos espacios cuyos habitantes comparten rasgos profesionales, religiosos, socioeconómicos o de identidades étnicas; es decir, que guardan cierta homogeneidad, y agrega que:

Para que pueda hablarse de un barrio es necesario un grado de frecuencia de proximidad a ciertos lugares, donde se encuentran los equipamientos urbanos, dentro de ciertos límites y en forma distintiva del resto de la ciudad, como condiciones de un comportamiento que sintetiza en el no ir más allá del mundo del peatón [haciendo referencia al término empleado por Raymond Ledrut]. (Gravano, 2005, p. 99).

Muchas de las distinciones anteriores - como podría ser la escala económica de un asentamiento obrero, la escala social de una agrupación minoritaria o el déficit de servicios públicos-, son las que le han valido a los barrios estigmas y prejuicios sobre seguridad, salubridad o desarrollo social; muchas de las ocasiones concebidos de manera previa o sin considerar que las problemáticas son producto de las circunstancias y el olvido de la sociedad en su conjunto, y no necesariamente de la comunidad que conforma al barrio.

Bajo las mismas condiciones de homogeneidad, la conjunción de un barrio también puede ser evidente en tanto el medio físico construido, ya sean viviendas de autoconstrucción, conjuntos multifamiliares -llamadas vecindades- o viviendas facilitadas por el Estado que, de acuerdo con el caso, pueden ser denominadas como de interés social o popular.

La vivienda de interés social para clases trabajadoras tiene que ser estudiada como un tipo de espacio local (o de vecindad) entre otros espacios, porque su valor relativo y su nivel de habitabilidad depende en buena medida de sus relaciones con el resto de la metrópoli [...] es un indicador elocuente de la manera en que distintos gobiernos han concebido a los sectores más necesitados y de la relación que han entretejido con ellos (Giglia, 2012, p. 104).

Si bien se ha establecido que estos no lo determinan, los elementos físicos dentro de un barrio auxilian a su comprensión y composición. Lynch (2008) llama a esto "continuidades temáticas", que bien pudieran manifestarse desde lo espacial; es decir, la topografía, la forma, las construcciones, el nivel 
de urbanización o el grado de mantenimiento, entre otros; o bien, como ya se ha hecho mención, a partir de la población y las actividades o peculiaridades sociales, culturales y/o económicas de esta.

En el texto clásico La arquitectura de la ciudad, Aldo Rossi (2017) relaciona íntimamente los términos área de estudio y barrio, puesto que ambos resultan un momento particular en el desarrollo de una ciudad, una abstracción espacial delimitada por elementos urbanos precisos, una muestra clínica que permite definir y analizar ciertos fenómenos y problemáticas urbanas. Francesc Muñoz (2008), reinterpreta las ideas de Rossi señalando que la ciudad debe comprenderse por medio de diferentes áreas de estudio, mismas que pudieran entenderse según los elementos de su urbanización o bien desde la propia historicidad del lugar.

Igualmente, los límites son de suma importancia en la descripción de un barrio. En este respecto, Jane Jacobs (2011) argumenta que el territorio es un sistema donde los integrantes de una banda se adueñan de las calles, viviendas o parques, impidiendo el paso a grupos de otros distritos. Si bien la autora urbana hace la connotación en términos de vandalismo, delincuencia y la seguridad que esto implica; entre los ciudadanos regulares esta práctica también es común en barrios consolidados donde la gente se apropia de los espacios públicos, comparten las tardes y conviven -aún si no cruzan una palabra entre ellos-, sintiéndose alarmados si un ente distinto a los cotidianos se presenta de manera hostil a romper con la frágil armonía.

De esta manera, la estructura física de un barrio puede ser tan distinta con respecto a otra como su propia vocación se lo permita. Territorialmente, podría ser definido por un elemento físico, visible y conocido (Jacobs, 2011); basta una gran avenida, un camellón, un parque vecinal, una plaza comercial, un edificio público, una iglesia o un simple muro para definir los límites de dicho barrio.

Ahora bien, la delimitación física de un barrio no plantea que este se desarrolle en lo individual e introspectivo, por el contrario, todo barrio resulta una pieza más en el engranaje que estructura una ciudad. Los barrios no resultan autosuficientes, estos dependen entre sí y de su capacidad de estar comunicados por una red de movilidad vial y cobertura de espacios públicos que inciten y faciliten el encuentro, procurando el fomento a la cohesión social; al igual que dependen de la dotación de equipamiento, infraestructura y servicios.

Yehoshua Cohen, en 1987, [...] brinda cuatro conceptos de contexto del barrio: el social, que apunta a las características de los individuos; el global, que se refiere a la reputación, los servicios, las relaciones con otros barrios; el urbano, que tiene relación con la ciudad o el sector de la misma; y el societal, que abarca a la sociedad mayor (Gravano, 2005, p. 111).

Hay que recordar que el barrio es una concepción social plasmada en lo territorial y no al revés, y que "incluso el ciudadano más urbanita se preocupa del ambiente de su calle y distrito, por muchas opciones que se le ofrezcan fuera del mismo. Y la mayoría de los vecinos dependen en gran manera de su barrio para su vida cotidiana" (Jacobs, 2011, p. 148).

Se concluye, entonces, que los barrios son una unidad básica donde se gesta la identidad de sus residentes, convirtiéndose en un lugar de mediana escala donde la gente y sus actividades cotidianas encuentran algo en común. Los barrios dan vida a la ciudad, mientras la ciudad los mantiene vivos a su vez, funcionando ambas como un organismo vivo donde sus sistemas conviven en un equilibrio entrópico. De la misma manera, la interrelación entre barrios es necesaria para que sus vocaciones tengan sentido; es decir, la razón de fortalecer a los barrios es también darle crédito al trabajo de planeación urbana al otorgar una correcta distribución de los usos de suelo, así como darle peso a la riqueza cultural que históricamente se han construido alrededor de cada barrio.

\section{Degradación Urbana}

Antes de continuar, es importante hacer notar que, dadas las connotaciones sociales que definen a un barrio, este requiere de tiempo para ser considerado como tal. Mientras que a un asentamiento habitacional cualquiera se le antepone el rótulo colonia, residencial o fraccionamiento al nombre otorgado; la denominación de barrio se adquiere mientras la comunidad crece y se arraiga, las vocaciones y límites se establecen, y el espacio físico envejece. El acelerado crecimiento de las ciudades medias, aunado a las contemporáneas políticas de producción de vivienda, impactan por igual a los barrios fundacionales, que pronto se encuentran sustituidos por los nuevos 
asentamientos; como los conjuntos habitacionales más recientes, que no obtienen el tiempo suficiente para envejecer como barrio.

Ahora, una vez establecidos los elementos que constituyen un barrio, será posible comprender las circunstancias en las que se presenta la etapa de degradación dentro de los ciclos de vida urbanos de los barrios.

De acuerdo con el área de estudio, la degradación puede entenderse $\mathrm{o}$ asociarse de diferentes maneras. Hasta ahora la connotación ha sido mayormente discutida desde una visión ecocentrista, donde se habla de la degradación medioambiental en los mismos términos que la deforestación, la conservación de la biodiversidad o el cambio climático.

En el recuento histórico y teórico que Pierri realiza acerca del término sustentabilidad, se menciona que "la degradación del medio ambiente está relacionado con los problemas sociales" (Pierri, 2005, p. 47). Por su parte, Enrique Leff emplea los términos degradación socioambientalydegradación ecológica como "problemas emergentes a escala planetaria" (Leff, 2011, p. 10). Incluso en el Reporte de la Comisión Mundial sobre el Medio Ambiente y Desarrollo: Nuestro futuro común (ONU, 1987), se enmarcan repetidamente las estrategias para mitigar la degradación medioambiental.

Si bien en los textos mencionados, la noción de degradación esta sobreentendida como la pérdida progresiva de valores, ninguno de ellos profundiza o aclara este concepto. Mucho menos construyen la idea a partir del medio ambiente urbano o con relación a los intereses culturales del ser humano. Recordemos que el medio ambiente urbano es parte del medio ambiente en general. Fernández (2010) señala que el hábitat natural del ser humano actual es la ciudad y agrega que ésta es resultado de una construcción social en un momento único, así como de la distribución espacial entre los habitantes y su convivencia. Por ello, y dado que la ciudad es una creación del ser humano moderno, el análisis de la degradación de un barrio debe ser entendida desde una postura antropocentrista.

Así, el término degradación se definirá como aquel momento en que las virtudes y cualidades de un elemento se van perdiendo. Un proceso paulatino donde aquello que se degrada reduce los elementos tangibles o intangibles que le dan valor hasta perder su utilidad. Para que un elemento llegue a degradarse, este debió tener primero cierta utilidad - utilidad para el ser humano-y después debió haber sido utilizado - utilizado por el ser humano-. Además, la degradación debió darse de manera progresiva y desde diversos procesos sociales. En caso de no cumplir con estos requerimientos, se estará hablando de otro tipo de fenómeno como de algo inerte, improductivo o accidental (Lynch, 2005).

\section{La Degradación como Parte de los Procesos Urbanos}

Comúnmente, el término utilizado para señalar el declive de un espacio urbano es deterioro. No obstante, este resulta insuficiente para explicar las problemáticas que van más allá de la descripción de un estado físico. Si bien el deterioro puede presentarse en cualquier espacio urbanizado, este no puede entenderse como sinónimo de la degradación, sino como un elemento que lo conforma.

En realidad, el deterioro es uno de los elementos que propician la degradación urbana pues, mientras el primer término involucra que un elemento físico pase a un peor estado o condición por causas naturales, como la propia caducidad del objeto o su prolongada exposición a la intemperie; el segundo, es un concepto antropocéntrico en donde además se involucra una decisión humana, como el uso excesivo del objeto o, por el contrario, la falta de mantenimiento y el abandono.

El deterioro puede detenerse, retrasarse o revertirse mediante la intervención del ser humano, pero si para este deja de tener interés, utilidad o valor, el mantenimiento no procede $y$, en su lugar, comienza el proceso de degradación. Por tanto, mientras el deterioro es un estado físico reversible, la degradación es un conjunto de fases que dependen del juicio social.

Lozano comenta que "de un modo paralelo al proceso de deterioro, es imaginable un proceso de ascenso o embellecimiento" (Lozano, 2019, p. 77), y por tanto el deterioro se convierte en un indicador o variable dentro de las agendas administrativas, con el fin de decidir sobre los procesos de renovación e intervención de las condiciones físicas y espaciales en la ciudad, según continua el mismo autor; sobre todo, cuando esto se relaciona con la contaminación visual y sus efectos en la imagen urbana (Sierra y Guevara, 2016). 
Por ello, la degradación de un barrio no solo se caracteriza por el deterioro de los elementos físicos o naturales; en dicho proceso también intervienen diversos aspectos sociales, incluyendo los económicos y políticos. Para algunos autores, la degradación puede reflejarse en las edificaciones a partir de:

Descensos significativos en el valor de las propiedades como consecuencia directa de la falta de mantenimiento y ausencia de nuevas inversiones en los bienes inmuebles privados, unido al abandono de las dotaciones en servicios sociales y bienes públicos ligados a los mismos (Cervelló y Segura, 2011, p. 35).

Ejemplifiquemos lo hasta aquí dicho, ver Figura 2. Si en un asentamiento habitacional cualquiera, un edificio es abandonado, este podría perder la pintura, encontrar sus aceras con polvo y basura, hundirse entre la maleza, inundarse entre goteras; o bien, podría ser vandalizado, saqueado, invadido, usado como basurero comunitario, empleado para llevar a cabo actos ilícitos, despojado de sus instalaciones e incluso incendiado. El acto humano interviene en ambos procesos, pero es la pérdida de los valores sociales, y no la intemperie, la que lo lleva a su degradación. Con ello, tanto el deterioro como la degradación agudizan la estigmatización preconcebida de los barrios, convirtiéndose en un lastre para la coordinación de los distintos intereses de los sectores gubernamentales, empresariales y de sociedad civil sobre el espacio urbano, lo que por ende desencadena un pausado desarrollo urbano.

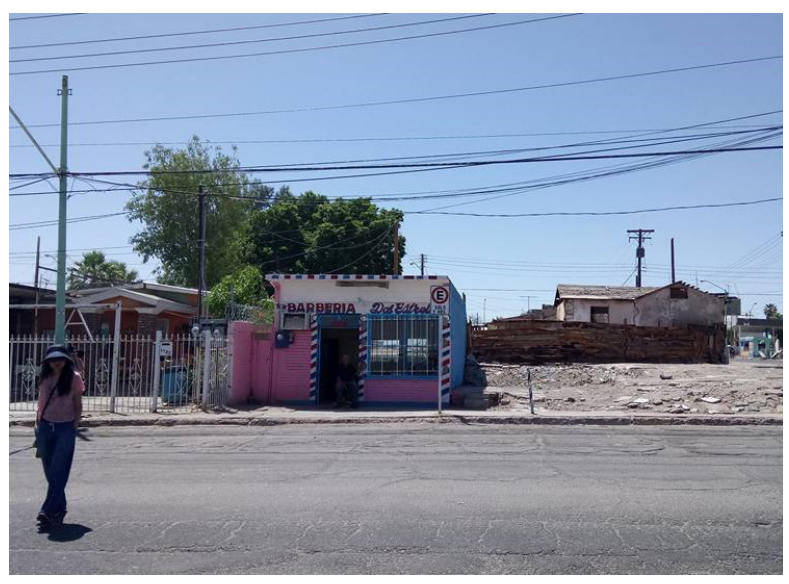

Figura 2. Barrio tradicional en proceso de degradación. Fuente: propia, 2017.
La degradación de un barrio puede detectarse con la presencia de elementos mesurables como el despoblamiento, la inactividad -social, recreativa, de intercambio económico u otros-, la vulnerabilidad delictiva, la desvalorización del suelo y, claro está, el deterioro de los elementos físicos. Esto concuerda con lo establecido por la Comisión Nacional de Vivienda (CONAVI) en México al editar la Guía para la redensificación habitacional en la ciudad interior, la cual señala que:

A través del tiempo los barrios en la ciudad interior experimentan los siguientes fenómenos: desvalorización, declinación, deterioro y degradación. Estos cuatro procesos son conceptualmente independientes y cada uno se refiere a fenómenos específicos, pero se encuentran íntimamente vinculados en su operación y suelen entrelazarse y reforzarse entre sí. [...] La degradación se refiere a la descomposición social que suele producirse como resultado de la interacción de los tres fenómenos anteriores [es decir: declinación, desvalorización y deterioro] (CONAVI, s/f, p. 12).

Por lo anterior, nuevamente se postula el que no solo el deterioro de los aspectos físicos determina la degradación, la intervención social tiene una gran injerencia en este proceso. Si se parte de los elementos requeridos para alcanzar la sustentabilidad social, como la satisfacción de las necesidades básicas, viabilidad de tener estabilidad económica, seguridad urbana y social, igualdad de oportunidades, calidad y estilo de vida elegido libremente, así como un sentido de pertenencia y arraigo, esto último en comunicación con las redes y morfología de la ciudad (Colantonio, 2007), la pérdida de estos elementos podría entenderse también como degradación.

Como resultado, la degradación también será posible conocerla a través de elementos cualitativos al vincularla con parámetros tales como la disminución de la cohesión social y del sentido de pertenencia, así como el aumento en la percepción de inseguridad, en tanto que el deterioro de los elementos físicos urbanos derive de una injerencia social. Así, pues, la degradación de un barrio involucra un proceso generacional donde se ven disminuidos los valores sociales, las responsabilidades compartidas y las prácticas tradicionales que conforman una comunidad; es decir, la merma de los atributos sociales. 
Una vez mesurados y conocidos los elementos que llevan a la degradación de un barrio, será posible contemplar la recuperación de este espacio. Y así como en arquitectura existe una clara diferenciación entre los grados de intervención en un edificio, desde la restauración hasta la remodelación, encontraremos que sobre la ciudad se puede actuar dependiendo de los objetivos e intenciones hacia un determinado espacio urbano. Hablemos, pues, de la rehabilitación urbana.

\section{Rehabilitación Urbana}

Ya que la degradación en las aceleradas ciudades contemporáneas es en muchas de las ocasiones prematura, como ya se hizo mención, la etapa de recuperación de los asentamientos habitacionales ha sido recurrente entre las nuevas inquietudes de la planeación urbana. La rehabilitación urbana o rehabilitación de barrios son términos empleados tanto en artículos científicos sobre planeación y urbanismo, como en planes y programas públicos nacionales e internacionales, para describir la intervención sostenible de espacios urbanos en degradación.

La definición e interpretación del concepto podría resultar distinta para cada caso en lo particular, derivando en diferentes aproximaciones de los objetivos primarios o, en el peor de los casos, impidiendo la realización de estos. Así, se presenta una noción del término con el fin de proponer un sustento válido durante la estructuración de estrategias urbano-sostenibles y proyectos de investigación relacionados.

La expresión rehabilitación pertenece al ámbito de la medicina, donde es entendida como un proceso de restauración integral para restablecer la capacidad de una persona, no solo en su condición física, también en la psicológica, social y económica (OMS, 1980). Trasladado al ejercicio de la planeación urbana, esto no tendría por qué cambiar su sentido ya que todo medio ambiente dañado, sea natural o construido, tiene la aptitud de ser rehabilitado a través de intervenciones oportunas y estratégicas a fin de restablecer su funcionalidad. En ocasiones dichos espacios no son capaces de continuar ejerciendo las actividades que originalmente los caracterizaron, sin embargo, logran adaptarse o transformarse a nuevos usos.
De acuerdo con organismos mexicanos, la rehabilitación "se orienta al mejoramiento de las condiciones físicas de las edificaciones existentes, en función de mejorar su adecuación a las funciones a que están destinadas [...] pueden realizarse a escala de edificios aislados o de barrio" (SEDESOL, s/f, p. 97). Fuera de América Latina, la rehabilitación también es un concepto clave a distintas escalas, como: viviendas, locales comerciales, infraestructura, barrios o centros históricos, tal como establece la Consejería de Vivienda y Ordenación del Territorio de la Junta de Andalucía, España, organismo que destaca en este tema por contar con un Plan Concertado de Vivienda y Suelo 2008-2012, en el cual la rehabilitación integral de barrios tiene el objetivo de:

Mejorar las condiciones de alojamiento de la población y otros aspectos de carácter urbanístico, social y económico, mediante la integración, la coordinación y el fomento de las distintas actuaciones que los agentes públicos y privados puedan desarrollar en dichos ámbitos urbanos (BOJA, 2008, p. 22).

Ahora bien, en algunos textos es posible encontrar estas atribuciones de recuperación de los espacios urbanos bajo otros conceptos, como: regeneración urbana o resiliencia urbana; en virtud de describir la capacidad que tiene un espacio de enfrentar y superar una dificultad por sí mismo.

En el Glosario de Términos de Desarrollo Urbano de SEDESOL en México se define regeneración urbana como un "proceso que al actuar sobre las causas generales y los factores específicos que dan origen al deterioro, contribuyen al desarrollo de las funciones, así como al mejoramiento de las condiciones del medio ambiente" (SEDESOL, $\mathrm{s} / \mathrm{f}, \mathrm{p}$. 92). Además, señala que las estrategias de regeneración urbana requieren ser integrales en los campos de lo físico y lo ambiental, pero sobre todo lo económico y lo social; y no una acción aislada que oculte los problemas urbanos.

Mientras que resiliencia urbana se describe como un entorno flexible, adaptable y sostenible; compuesto por una red de sistemas físicos -calles, edificios, infraestructura en comunicaciones, instalaciones eléctricas e hídricas, entre otrosque deben ser capaces de resistir y funcionar bajo tensiones extremas y así evitar ser vulnerable ante cualquier tipo de desastre (Godschalk, 2003). 
La rehabilitación a nivel barrial podría abordarse a partir de los componentes físicos, lo que incluye el adaptar los usos de suelo, redistribuir la densidad poblacional, optimizar los flujos de traslado y reactivarlossubcentros consolidados. Esto brindaría un uso eficiente del equipamiento y otros servicios otorgados, un mayor control en la administración de los recursos, así como la permanencia de las áreas de amortiguamiento o reservas territoriales. En materia económica, esto podría propiciar una serie de ventajas, como la atracción de inversores, la generación de empleos directos e indirectos, la reducción de costo y tiempo en el traslado de las mercancías o la promoción turística a partir de una mayor diversidad de actividades y amenidades.

Empero, las grandes inversiones de proyectos urbanísticos y arquitectónicos también podrían ocasionar desventajas sociales, ya que "la rehabilitación urbana también incentiva procesos de especulación que a su vez engendran desplazamientos de la población con menores recursos" (Boivin, 2013, p. 3), generando un fenómeno de gentrificación. El fenómeno urbano conocido como gentrificación, es descrito como "el proceso de sustitución de las clases obreras por clases medias-altas en zonas degradadas y abandonadas de la ciudad" (Boivin, 2013, p. 2).

Desde una postura crítica es posible comprender que estos fenómenos se susciten en un territorio y se exhiban en la atención que se le presta a un barrio con respecto a otro; entendiendo que la tensión entre la acumulación del capital, la institución política que lo regula y la resistencia social, cobran especial interés en la formación de las ciudades. No obstante, al considerar las implicaciones que conciben a un barrio, siempre valdrá la pena procurar el rescate de la comunidad que lo constituye. El valor histórico que obtiene a lo largo de su conformación, las victorias sociales que se obtuvieron a partir de los movimientos de resistencia; las leyendas y los personajes urbanos que se mitificaron por generaciones; los inmuebles, servicios, infraestructura y demás componentes físicos de la urbanización que se consolidaron con los años, simplemente son elementos irremplazables.

Bajo la primicia de impulsar y apoyar estrategias de planeación urbana encaminadas al desarrollo social sostenible, la rehabilitación urbana deberá vincularse con la recuperación y revalorización del patrimonio histórico y social que caracteriza a los barrios, y requiere de la participación de la ciudadanía, elementos que concuerdan con los objetivos de la sustentabilidad social.

\section{Pensar las Estrategias de Rehabilitación}

Una vez definido el marco de entendimiento del bucle que se forma entre la degradación de barrios y la rehabilitación urbana, dentro del estudio de la evolución de ciudades, se podrá continuar con el establecimiento de estrategias técnicas y normativas de la planeación urbana, adecuadas y contextualizadas a las condiciones de cada área de estudio.

Entre las recomendaciones más reconocidas, opuestas a la hegemonía de la planeación nacional ineficiente, Jacobs (2011) declara que el objetivo principal de la urbanización debería ser el impulso de la diversidad urbana con calles interesantes que, junto con plazas, parques y edificios públicos, conformen una red continua y distritos con vocación definida. De esta manera, se recomienda el que las ciudades contemplen una mayor organización para promover esta distinción entre barrios. La idea de impulsar la vocación de los barrios se vincula con aceptar la existencia de ellos como un hecho social inherente a la vida en ciudad, de conocer a la comunidad que lo integra y estar al tanto de sus necesidades.

Para lograr lo anterior, sería necesario un adecuado diseño urbano, en el cual se contemple la conjunción de cuatro condiciones que trabajen al unísono: la combinación de usos de suelo primarios que detonen los secundarios, el fraccionamiento de pequeñas manzanas para propiciar trayectos cortos y mayor número de esquinas, la inclusión de edificios antiguos que minimicen los altos costos de compra o renta, así como la necesidad de aumentar la concentración de habitantes fijos y visitantes y usuarios flotantes. Si una de ellas faltase se frustraría el potencial de la zona a rehabilitar (Jacobs, 2011).

Cabe decir que, para Jacobs (2011), la viabilidad de dichas condiciones depende de cada ciudad, pues lo que funciona en un sector puede fallar en otro; además, su implementación no garantiza el éxito de la rehabilitación urbana. La intención primaria de estos recursos es atraer, aumentar, concentrar y mantener la densidad poblacional en las calles de una ciudad, lograr esto es la verdadera rehabilitación. Con esto se puede concluir que la mejor estrategia es la prevención; 
mantener un sistema urbano sano, donde todos sus sectores trabajen conjuntamente, donde los actores primarios y secundarios no inciten la segregación; y donde no exista un desarrollo urbano desequilibrado, desigual e insostenible, ver Figura 3.

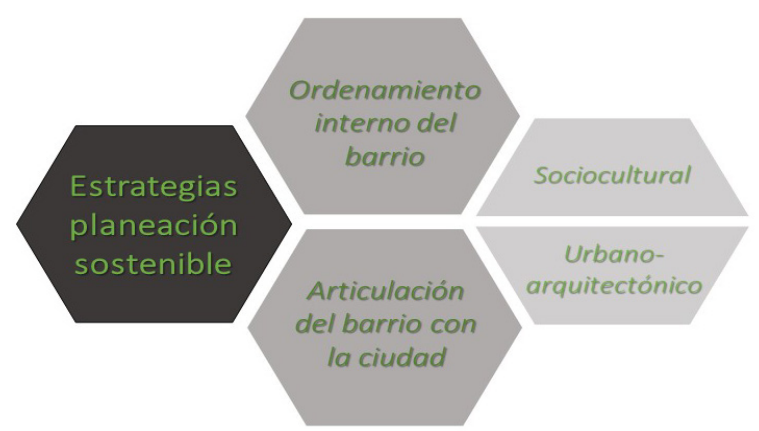

Figura 3. Diagrama de estrategias para una rehabilitación urbana sostenible.

Fuente: elaboración propia con base en investigación documental, 2021.

\section{Reflexiones Finales}

Si la degradación involucra aspectos de trascendencia social, en la misma medida que el deterioro urbano, los planes de recuperación urbana deben promover un enfoque con las mismas proporciones entre la regeneración comunitaria y la renovación urbana. Sin embargo, resulta común y cómodo atender inequívocamente los baches en las calles, la luminaria en los postes o la pintura en los cordones de las banquetas, tachando de subversivas las inquietudes sobre el incumplimiento de las responsabilidades conjuntas.
Como se hizo mención, la planeación urbana en la América Latina contemporánea resultan en su mayoría tendenciosas a favorecer un solo ámbito o una idea, cuando debería ser imparcial, excluyen a los integrantes de la comunidad y niegan los valores que han compuesto como barrio, imperando entonces los intereses hegemónicos tan a menudo citados y normalmente conocidos.

Como estrategia para los estudios preliminares y factibilidad de operación dentro de la planeación urbana sostenible, previa a la intervención, es recomendable como punto de partida la definición conceptual del perímetro en cuestión y las problemáticas en ésta. ¿Es acaso un barrio? Con todas las connotaciones temporales, culturales, de virtud o de infamia que involucra, ¿Es acaso un barrio en deterioro? De ser así, cuáles son los factores externos involucrados, ¿Es acaso un barrio en degradación? Y de ser así, cuáles son los valores perdidos.

Atendiendo lo anterior, sería posible enfocar el análisis y organizar los esfuerzos, en planes parciales que brinden continuidad y certidumbre para la comunidad; donde se rescaten los elementos tangibles e intangibles, históricos y contemporáneos; los cuales fortalezcan los lazos de la comunidad, de manera interna y con respecto a su ciudad. Que tome en consideración la movilidad, la autonomía, la seguridad, la vivienda, la conectividad, la relación entre actividades, la diversidad y la cohesión. En otras palabras: estrategias para el ordenamiento interno del barrio, aunado a estrategias para la articulación del barrio con la ciudad.

\section{REFERENCIAS}

Amérigo, M. y Pérez López, R. (2010). Ambientes residenciales. En; J. I. Aragonés y M. Amérigo (Coord.), Psicología ambiental. (pp. 163-182). Madrid, España: Pirámide.

Boivin, R. R. (2013). Rehabilitación urbana y gentrificación en el barrio de Chueca: la contribución gay. Revista Latino-americana de Geografia e Gênero, 4 (1), pp. 114-124. doi: 10.5212/Rlagg.v.4.i1.3068

BOJA (Boletín Oficial de la Junta de Andalucía) (2008). Decreto 395/2008, de 24 de junio, por el que se aprueba el Plan Concertado de Vivienda y Suelo 2008-2012. Sevilla, España: Junta de Andalucía. Recuperado de: https://www.juntadeandalucia.es/boja/2008/130/2

Castro, L. J. (2018). Introducción. En L. J. Castro (Coord.), La planeación sostenible de ciudades. Propuestas para el desarrollo de infraestructura (pp. 9-14) Ciudad de México, México: Fondo de Cultura Económica. 
Cervelló Royo, R. y Segura, B. (2011). Efectos de las intervenciones públicas en regeneración urbana: un modelo para evaluar su eficiencia. Revista de Economía Pública, Social y Cooperativa, (70), pp. 33-54. Recuperado de: http://ciriec-revistaeconomia.es/es/revista/?num=2643

Charry, C. A. (2006). Perspectivas conceptuales sobre la ciudad y la vida urbana: el problema de la interpretación de la cultura en contextos urbanos. Antipoda, (2), pp. 209-228. doi: 10.7440/antipoda2.2006.11

Choay, F. (2019). Alegoría del patrimonio. Barcelona, España: Gustavo Gili.

Colantonio, A. (2007). Social sustainability: an exploratory analysis of its definition, assessment methods, metrics and too/s. (Documento de trabajo núm. 2007/1). Oxford, Reino Unido: Oxford Brooks University. Recuperado de: http://oisd.brookes.ac.uk/sustainable communities/resources/SocialSustainability Metrics and Tools.pdf

CONAVI (Comisión Nacional de Vivienda) (s/f). Guía para la redensificación habitacional en la ciudad interior. Ciudad de México, México: Gobierno Federal, SEDESOL, CONOREVI, UNAM, FOVISSSTE, INFONAVIT, SHF, CONAVI. Recuperado de: https://www.cmic.org.mx/comisiones/sectoriales/vivienda/biblioteca/ archivos/guiaRedensificacionHabitacional.pdf

Dave, S. (2011). Neighbourhood density and social sustainability in cities of developing countries. Sustainable Development, 19 (3), pp. 189-205. Recuperado de: https://onlinelibrary.wiley.com/action/ $\underline{\text { doSearch?target=default\&ContribAuthorStored =Dave,\%20Seema\&SeriesKey }=10991719}$

Delgado Viñas, C. (2016). Miradas sobre la ciudad desde la geografía, la historia y el urbanismo. El estado de la cuestión a comienzos del siglo XXI. Ciudades, 19 (1), pp. 117-142. Recuperado de: http://iuu.uva.es/ REVISTA/Ciudades\%2019/Ciudades\%2019\%20117-142\%20DELGADO\%20VINAS.pdf

Fernández, B. (2010). El medio urbano. En: J. I. Aragonés y M. Amérigo (Coords.), Psicología ambiental (pp. 241-260). Madrid, España: Pirámide.

Gravano, A. (2005), El barrio en la teoría social. Buenos Aires, Argentina: Espacio Editorial.

Giglia, A. (2012). El habitar y la cultura. Perspectivas teóricas y de investigación. Barcelona: Anthropos Editorial y Universidad Autónoma Metropolitana.

Godschalk, D. R. (2003). Urban Hazard mitigation: creating resilient cities. Natural Hazards Review, 4 (3), pp. 136-143. doi: 10.1061/(ASCE)1527-6988(2003)4:3(136)

Jacobs, J. (2011). Muerte y vida de las grandes ciudades. Navarra, España: Capitán Swing Libros, S.L.

Lamy, B. (2006). Sociología urbana o sociología de lo urbano. Estudios demográficos y urbanos, 21 (1), pp. 211-225. doi: 10.24201/edu.v21i1.1267

Leff, E. (2011). Sustentabilidad y racionalidad ambiental: hacia 'otro' programa de sociología ambiental. Revista mexicana de sociología, 73 (1), pp. 5-46. Recuperado de: http://www.redalyc.org/articulo. oa?id=32118437001

Lozano Rivera, C. (2019). El deterioro como concepto y criterio de renovación urbana. En E. Aguirre Ramírez, E., C. Lozano Rivera \& V. Mejía Amézquita (Comp.). Deterioro, obsolescencia y configuración urbana. Reflexiones y manifestaciones (pp. 67-83) México: UACJ \& UABC.

Lynch, K. (2005). Echar a perder. Un análisis del deterioro. Barcelona, España: Gustavo Gili. 
Vizcarra - De la Degradación y la Rehabilitación: Apuntes Conceptuales en torno a la Noción de Barrio

Lynch, K. (2008), La imagen de la ciudad. Barcelona, España: Gustavo Gili.

Muñoz, F. (2008). Urbanización. Paisajes comunes, lugares globales. Barcelona, España: Gustavo Gili.

OMS (Organización Mundial de la Salud) (1980). Rehabilitation. Regional Committee For The Eastern Mediterranean (Thirtieth Session: Agenda item 11) WHO.

ONU (Organización de las Naciones Unidas) (1987). Report of the World Commission on Environment and Development: Our Common Future, UN. Recuperado de: https://sustainabledevelopment.un.org/ content/documents/5987our-common-future.pdf

Pérgolis-Valsecchi, J.C., \& Quijano-Gómez, E. (2020). Memoria, patrimonio, consumo y olvido. Procesos Urbanos, 7 (2). https://doi.org/10.21892/2422085X.510

Pierri, N. (2005). Capítulo 2. Historia del concepto de desarrollo sustentable. En: G. Foladori y N. Pierri (Coord.), ¿Sustentabilidad? Desacuerdos sobre el desarrollo sustentable (pp. 27-82). México D.F.: Universidad Autónoma de Zacatecas y Miguel Ángel Porrúa.

Rizo, M. (2006). Conceptos para pensar lo urbano: el abordaje de la ciudad desde la identidad, el habitus y las representaciones sociales. Bifurcaciones, (6), pp. 1-13. Recuperado de: http://www.bifurcaciones. cl/2006/03/conceptos-para-pensar-lo-urbano/

Rojas, R. I.; Peña, C. y Corona, E. A. (2012). Marco para la evaluación y planeación de la sustentabilidad urbana: Zona metropolitana de Tijuana-Rosarito-Tecate. En A. Ranfla González y L. M. Ortega Villa (Coord.), Procesos urbanos en Baja California: análisis, planeación y sustentabilidad (pp. 185-220). Mexicali, México: Universidad Autónoma de Baja california y Red Nacional de Investigación Urbana.

Rossi, A. (2017). La arquitectura de la ciudad. Barcelona, España: Gustavo Gili.

SEDESOL (Secretaría de Desarrollo Social) (s/f). Glosario de Términos de Desarrollo Urbano. México: SEDESOL. Recuperado de: https://es.scribd.com/document/166114774/Glosario2000sedesol-determinos-de-desarrollo-urbano

Sierra Franco A., \& Guevara Guevara O. (2016). Contaminación visual en centros históricos: un problema estético y de valor social de las ciudades actuales. Procesos Urbanos, 3 (3), $133-142$. https://doi. org/10.21892/2422085X.273 